\title{
In vitro fermentation of carbohydrate by breast fed and formula fed infants
}

\author{
A M Parrett, C A Edwards
}

\begin{abstract}
Unabsorbed carbohydrates are fermented by colonic bacteria to short chain fatty acids (SCFA) which are rapidly absorbed, salvaging energy and reducing stool output. There are marked differences between the faecal flora and SCFA of breast fed (BF) and formula fed (FF) infants which may be related to the higher incidence of diarrhoea in FF infants. Part of this effect may be caused by a difference in the ability of the microflora to ferment carbohydrate. To test the hypothesis that BF and FF have different fermentation capacities for simple and complex carbohydrates, in vitro cultures of faeces from healthy infants (2-10 weeks; $11 \mathrm{BF}, 11 \mathrm{FF}$ ) containing glucose, lactose, raftilose (a fructo-oligosaccharide), or soybean polysaccharide were incubated anaerobically. Results were compared with those of adult faecal cultures using the same carbohydrates. Cultures of faeces from BF and FF infants produced comparable amounts of total SCFA in all cultures. These cultures produced less SCFA than those from adult faeces and produced very little SCFA from complex carbohydrate. BF cultures produced more acetic acid than FF in all cultures, whereas FF cultures produced more propionate with sugars and more butyrate with raftilose. Both groups of infants produced less butyrate than adults in all cultures. Thus it is unlikely that a lower ability to ferment carbohydrate is a major cause of increased risk of diarrhoea in FF fed infants but individual SCFA production may be important.

(Arch Dis Child 1997;76:249-253)
\end{abstract}

Keywords: fermentation; carbohydrate.

Breast fed infants are less likely to suffer infectious diarrhoea than infants fed modern infant formula. ${ }^{1}$ This may be due to differences in their colonic bacterial flora and its metabolic activity. Bifidobacteria and lactobacilli dominate the stools of breast fed infants, whereas formula fed infants have more enterobacteria, streptococci, and bacteroides species ${ }^{2}$ in their stools. These differences are reflected in the profile of faecal short chain fatty acids (SCFA) which result from the fermentation of carbohydrate in the colon. We have previously shown that the faeces of breast fed infants contain mainly acetic and lactic acid whereas those of formula fed infants have acetic and propionic acid with small amounts of butyric acid. ${ }^{3}$

Faecal SCFA profiles are the end result of both production and absorption and may not represent events in the proximal colon, where most fermentation occurs. They give very little information about the ability of infants to ferment carbohydrate. It may be the ability of the colonic flora to ferment carbohydrate, and the absorption of the resultant SCFA which promote water absorption, that helps prevent diarrhoea.

It is difficult to study colonic fermentation in vivo. In vitro studies using faecal bacteria to inoculate cultures containing different carbohydrates have proved useful models of fermentation in adults. ${ }^{4}$ Very few studies of this kind have been carried out using faecal bacteria from infants and these have measured fermentation of endogenous substrates or added sugars. $^{56}$

Lifshitz et $a l^{6}$ compared the in vitro fermentation of lactose by faecal bacteria from breast fed and formula fed infants at $\mathrm{pH} 6.8$ and $\mathrm{pH}$ 5.5. At low $\mathrm{pH}$ the cultures of faeces from breast fed infants showed less hydrolysis of lactose into monosaccharides. In cultures of faeces from formula fed infants, however, lactose hydrolysis was maintained, whereas fermentation was inhibited by $25 \%$. This resulted in an increased osmolality due to unfermented monosaccharides, which, if it occurred in vivo, would increase risk of diarrhoea. These results suggested that the faecal flora of breast fed and formula fed infants had different metabolic characteristics which were influenced by $\mathrm{pH}$ in different ways. These cultures were maintained at constant $\mathrm{pH}$ and were incubated for only one hour. Fermentation occurs over a much longer time in vivo and differences seen initially may be overcome before colonic function is adversely affected.

There may be a greater influence of the different colonic microflora of breast fed and formula fed infants on their ability to ferment oligosaccharides and complex carbohydrates. In this study we employed a simple in vitro method to test the hypothesis that the difference in the faecal flora of breast fed and formula fed infants might influence their ability to ferment carbohydrates. If formula fed infants have a lower fermentation capacity this may be a contributing factor to their greater risk of diarrhoea. In addition, this model would confirm whether the differences in SCFA patterns seen in the faeces of breast fed and 
Table 1 Comparison of $p H$ values in breast fed infants, formula fed infants, and adults after in vitro incubation of faecal bacterial cultures containing various simple and complex carbohydrates as substrates

\begin{tabular}{|c|c|c|c|c|c|c|}
\hline & \multicolumn{2}{|c|}{$\begin{array}{l}\text { Breast fed infants } \\
(n=11)\end{array}$} & \multicolumn{2}{|c|}{$\begin{array}{l}\text { Formula fed infants } \\
(n=11)\end{array}$} & \multicolumn{2}{|c|}{ Adults $(n=6)$} \\
\hline & Median & Range & Median & Range & Median & Range \\
\hline Blank & 7.0 & $5.5-7.6$ & 7.0 & $6.8-7.5$ & 7.1 & $7.0-7.3$ \\
\hline Glucose & 4.3 & $3.5-6.3$ & 5.1 & $3.6-6.3$ & 4.9 & $4.3-5.7$ \\
\hline Lactose & 4.3 & $3.5-5.8$ & 5.0 & $3.7-6.3$ & ND & ND \\
\hline Raftilose & 5.0 & $3.8-5.7$ & 5.0 & $4.2-5.9$ & 4.7 & $4.4-5.4$ \\
\hline \multicolumn{7}{|l|}{ Soybean } \\
\hline polysaccharide & $6.7^{\star}$ & $5.3-7.1$ & $6.6^{\star}$ & $6.3-7.0$ & 6.3 & $5.9-6.5$ \\
\hline
\end{tabular}

formula fed infants ${ }^{3}$ are due to differences in production or absorption.

\section{Methods}

SUBJECTS

Twenty two full term healthy infants were recruited by advertisement and from the postnatal wards at the Queen Mother's Maternity Hospital, Glasgow. None of these infants, nor any of the breast feeding mothers, had received any antibiotics at the time of recruitment or at any time before giving stool samples.

Fresh faeces (processed within one hour of passage) were obtained from exclusively breast fed infants (seven males, four females), aged 2-13 weeks (median 5 weeks) and exclusively formula fed infants (one male, 10 females) aged 2-10 weeks (median 6 weeks). Infants who received mixed feeding with breast and formula milk, or who had received food other than milk, were excluded from the study. Formula fed infants were receiving one of several standard cows' milk based formulas (Ostermilk, Ostermilk 2, SMA Gold Cap, SMA White Cap).

FERMENTATION MODEL

Faecal samples were collected from the infants at their homes and were processed within one hour of defecation. After weighing, a faecal slurry (32\% wt/vol) was made with prereduced phosphate buffer, $\mathrm{pH}$ 7. One millilitre of this was used to inoculate $9 \mathrm{ml}$ prereduced basic salts medium, ${ }^{4}$ containing $100 \mathrm{mg}$ of carbohydrate substrate: glucose $(\mathrm{BDH})$, lactose (Sigma), raftilose (Raffinerie Tirlemontoise SA), or soybean polysaccharide (Scientific Hospital Supplies). Cultures contained a total volume of $10 \mathrm{ml}$. If less faecal material was available amounts were halved to give a total culture volume of $5 \mathrm{ml}$. Cultures were incubated in an anaerobic jar at $37^{\circ} \mathrm{C}$. A control culture without carbohydrate was also incubated to estimate the SCFA produced from endogenous carbohydrate, mucin, or other substrates in the original faecal sample.

\section{CARBOHYDRATE SUBSTRATES}

The carbohydrate substrates were chosen for specific reasons. Most bacteria can ferment glucose which is the main constituent sugar of cellulose, resistant starch, and maltodextrins. Lactose is the main carbohydrate in milk. Raftilose is an example of fructo-oligosaccharides which are present in significant quantities in human milk. Soybean polysaccha- ride contains cellulose, hemicellulose, and soluble fibre and is representative of similar dietary fibre sources in the human diet. Many commercial weaning foods contain soya flour.

\section{MEASUREMENTS}

After 24 hours, the $\mathrm{pH}$ of the fermentation fluid was measured before storage at $-20^{\circ} \mathrm{C}$ for later analysis of SCFA and lactic acid content. SCFA were measured by gas liquid chromatography of acidified ether extracts using $\beta$ methyl valeric acid as an internal standard. ${ }^{7}$ Lactic acid was measured similarly after methylation with sulphuric acid and methanol, using succinic acid as an internal standard. ${ }^{8}$

\section{FAECAL ANALYSIS}

Where sample size was sufficient $(\mathrm{n}=20)$, aliquots of faeces were weighed, brought to $\mathrm{pH}$ $>9$ with sodium hydroxide to prevent loss of SCFA, frozen, and then freeze dried for calculation of faecal water and analysis of faecal SCFA.

\section{STATISTICS}

Results from cultures of faeces from breast fed infants, formula fed infants, and adults were compared by Mann-Whitney U test after subtraction of the no carbohydrate blank to allow for SCFA produced from other substrates in faeces.

ETHICS APPROVAL

This study was approved by the ethics committee of the Yorkhill NHS Trust. The mothers of all infants taking part gave written informed consent.

\section{Results}

$\mathrm{pH}$

There were no significant differences in the final $\mathrm{pH}$ between the two infant feeding groups for any of the substrates (table 1). The greatest fall in $\mathrm{pH}$ after 24 hours of incubation was observed for the sugars and fructooligosaccharide.

\section{SCFA CONCENTRATION}

Total SCFA production was similar for breast fed and formula fed infants for all substrates (table 2). Cultures of faeces from both groups of infants, however, produced much less SCFA than cultures of adult faeces. In the control culture (no carbohydrate), significantly less total SCFA were produced by the formula fed baby group $(p<0.05)$ than by the breast fed infants.

Cultures of faeces from breast fed infants produced more acetic acid from all substrates than those from formula fed infants and adults. Significantly more propionic acid was produced by the formula fed group with glucose, lactose, raftilose, and no carbohydrate, and more n-butyric acid was produced by the formula fed group with glucose and raftilose, compared with the breast fed group. Formula fed infants had SCFA profiles much closer to those produced by cultures of adult faeces. 
Table 2 Short chain fatty acid (SCFA) concentrations ( $\mu \mathrm{mol} / \mathrm{ml}$ ) of breast fed infants, formula fed infants, and adults after in vitro incubations of faecal bacterial cultures containing simple and complex carbohydrates as substrates

\begin{tabular}{|c|c|c|c|c|c|c|}
\hline & \multicolumn{6}{|c|}{ SCFA concentration $(\mu \mathrm{mol} / \mathrm{ml})$} \\
\hline & \multicolumn{2}{|c|}{ Breast fed infants $(n=11)$} & \multicolumn{2}{|c|}{ Formula fed infants $(n=11)$} & \multicolumn{2}{|c|}{ Adults $(n=6)$} \\
\hline & Median & Range & Median & Range & Median & Range \\
\hline \multicolumn{7}{|l|}{ Total SCFA } \\
\hline Blank & 18.15 & $5.6-28.0$ & $10.2^{\star}$ & $0.9-19.1$ & 25.4 & $14.1-60.6$ \\
\hline Glucose & $55.6 \dagger$ & $0.0-77.6$ & $48.6^{\star}$ & $8.0-100.9$ & 87.4 & $66.4-107.9$ \\
\hline Lactose & $44.6^{\circ}$ & $0.0-63.0$ & 40.9 & $21.9-78.7$ & ND & ND \\
\hline Raftilose & $33.7 \dagger$ & $5.9-48.9$ & $42.7^{\star}$ & $10.1-125.9$ & 74.4 & $49.0-123.1$ \\
\hline Soybean polysaccharide & $12.6 \dagger$ & $8.1-40.2$ & $14.1 \dagger$ & $0.0-69.4$ & 64.2 & $35.1-119.1$ \\
\hline \multicolumn{7}{|l|}{ Acetic acid } \\
\hline Blank & $13.1 \S$ & $3.6-19.2$ & 6.7 & $0-12.3$ & 9.8 & $5.2-15.3$ \\
\hline Glucose & 62.5 & $0.6-79.2$ & 27.1 & $3.6-72.1$ & 56.6 & $42.7-75.0$ \\
\hline Lactose & 46.7 & $0-67.9$ & 30.1 & $6.9-79.2$ & ND & ND \\
\hline Raftilose & $30.4^{\star}$ & $8.1-42.9$ & $30.4^{\star}$ & $4.6-75.8$ & 39.1 & $33.3-95.6$ \\
\hline Soybean polysaccharide & $9.6 \dagger$ & $6.0-36.5$ & $7.7^{\star}$ & $0-38.8$ & 34.3 & $14.8-72.7$ \\
\hline \multicolumn{7}{|l|}{ Propionic acid } \\
\hline Blank & $3.7 \S$ & $1.7-8.0$ & $2.2^{\star}$ & $0.7-5.4$ & 4.2 & $2.0-14.9$ \\
\hline Glucose & $0 \ddagger \|$ & $0-6.0$ & 9.5 & $1.4-36.2$ & 16.1 & $7.7-18.8$ \\
\hline Lactose & $0 \|$ & $0-7.0$ & 11.1 & $1.4-17.6$ & ND & ND \\
\hline Raftilose & $1+9$ & $0-8.4$ & 6.9 & $1.4-37.9$ & 12.4 & $3.7-19.3$ \\
\hline Soybean polysaccharide & $2.9 \dagger$ & $1.5-5.6$ & $4.6 \dagger$ & $0-19.6$ & 15.1 & $8.6-19.1$ \\
\hline \multicolumn{7}{|l|}{ Butyric acid } \\
\hline Blank & $0.18 \dagger$ & $0-5.1$ & $0.6 \dagger$ & $0-1.4$ & 2.6 & $1.4-4.5$ \\
\hline Glucose & $0+5$ & $0-13.9$ & $0.4^{\star}$ & $0-10.8$ & 6.5 & $1.1-32.4$ \\
\hline Lactose & 0 & $0-2.7$ & 0.6 & $0-6.8$ & ND & ND \\
\hline Raftilose & $0 \star 8$ & $0-11.4$ & 4.0 & $0-28.9$ & 11.2 & $1.6-28.8$ \\
\hline Soybean polysaccharide & $0.3 \dagger$ & $0-5.8$ & $0.6^{\star}$ & $0-6.5$ & 6.0 & $3.5-20.8$ \\
\hline \multicolumn{7}{|l|}{ Lactic acid } \\
\hline Blank & $0^{\star}$ & $0-2.6$ & 0 & $0-0$ & 0.7 & $0-11.6$ \\
\hline Glucose & 25.4 & $10.0-47$ & 8.6 & $0-58.9$ & 25.1 & $0-45.3$ \\
\hline Lactose & 30.3 & $10.8-56.4$ & 21 & $0-82.2$ & ND & ND \\
\hline Raftilose & 5.9 & $0-27.4$ & 3.4 & $0-158.5$ & 17.6 & $0-35.7$ \\
\hline Soybean polysaccharide & 0 & $0-3.2$ & 0 & $0-0.6$ & 2.0 & $0-16.9$ \\
\hline
\end{tabular}

$\star \mathrm{p}<0.05 ; \dagger \mathrm{p}<0.01 ; \ddagger \mathrm{p}<0.001 v$ adults.

p $<0.05 ; \uparrow p<0.01 ; \| \mathrm{p}<0.001 v$ formula fed infants.

$\mathrm{ND}=$ not done.

Cultures of faeces from breast fed infants had significantly less propionic and butyric acid than adult cultures for all substrates tested (table 2).

MOLAR PROPORTIONS

The breast fed infants had significantly larger molar proportions of acetic acid with all substrates and also with the no carbohydrate blank control (table 3). Formula fed infants had significantly larger proportions of propionic acid with glucose and lactose but when raftilose was the substrate, it was n-butyric acid production that was increased. There were no significant differences in the molar proportions of lactic acid between the two groups of infants.

LACTIC ACID

Although there was no significant difference between the amount of lactic acid produced by the two groups with all substrates, there was a trend for greater lactic acid production by the breast fed infants. More cultures of faeces from breast fed infants produced large amounts of

Table 3 Proportions of individual SCFA (as a percentage of total SCFA) produced by in vitro cultures of faecal bacteria from breast fed infants, formula fed infants, and adults containing simple and complex carbohydrates

\begin{tabular}{|c|c|c|c|c|c|c|}
\hline & \multicolumn{2}{|c|}{ Breast fed infants $(n=11)$} & \multicolumn{2}{|c|}{ Formula fed infants $(n=11)$} & \multicolumn{2}{|c|}{ Adults $(n=6)$} \\
\hline & Median & Range & Median & Range & Median & Range \\
\hline \multicolumn{7}{|l|}{ Acetic acid } \\
\hline Blank & $71.6+\$$ & $54.6-83.8$ & $59.0^{\star}$ & $0-71.9$ & 40.8 & $24.6-56.8$ \\
\hline Glucose & 98.0 ᄎब & $56.2-99.7$ & 68.3 & $44.5-89.6$ & 66.7 & $52.1-88.7$ \\
\hline Raftilose & 87.6 ᄎ & $56.4-98.7$ & 62.0 & $39.1-85.8$ & 63.1 & $47.4-86.9$ \\
\hline Soybean polysaccharide & $73.7+\$$ & $54.5-85.9$ & 56.6 & $0-72.6$ & 55.3 & $42.2-61.0$ \\
\hline \multicolumn{7}{|l|}{ Propionic acid } \\
\hline Blank & 21.6 & $13.0-33.4$ & 19.7 & $6.8-73.1$ & 14.9 & $7.1-52.1$ \\
\hline Glucose & $1.4^{\star} \|$ & $0.0-24.4$ & 26.3 & $8.6-43.6$ & 17.6 & $8.8-26.7$ \\
\hline Raftilose & 11.9 & $0.8-19.0$ & 16.4 & $9.2-59.6$ & 14.9 & $4.0-27.9$ \\
\hline Soybean polysaccharide & 19.2 & $11.7-36.3$ & 28.8 & $0.0-45.7$ & 23.2 & $14.6-32.5$ \\
\hline \multicolumn{7}{|l|}{$N$-butyric acid } \\
\hline Blank & $0.9^{\star}$ & $0.0-18.4$ & $4.4 \dagger$ & $0.0-8.2$ & 11.2 & $5.0-18.1$ \\
\hline Glucose & $0.2^{\star}$ & $0.0-28.1$ & $2.9^{\circ}$ & $0.1-14.3$ & 8.9 & $1.4-30.0$ \\
\hline Raftilose & $0.2 \star \sqrt{\zeta}$ & $0.0-24.8$ & 9.3 & $0.5-34.1$ & 13.5 & $1.8-36.9$ \\
\hline Soybean polysaccharide & $0.4^{\star}$ & $0.0-20.6$ & $4.0^{\star}$ & $0.0-17.0$ & 10.9 & $7.4-18.9$ \\
\hline \multicolumn{7}{|l|}{ Lactic acid } \\
\hline Blank & $0.0^{\star}$ & $0.0-8.4$ & 0.0 & $0.0-0.0$ & 3.2 & $0.0-28.0$ \\
\hline Glucose & 25.8 & $9.6-60.4$ & 13.3 & $0.0-77.7$ & 24.7 & $0.0-35.5$ \\
\hline Raftilose & 10.3 & $0.0-44.1$ & 3.2 & $0.0-88.1$ & 15.9 & $0.0-37.3$ \\
\hline Soybean polysaccharide & 0.0 & $0.0-8.5$ & 0.0 & $0.0-1.3$ & 3.8 & $0.0-30.6$ \\
\hline
\end{tabular}

$\star \mathrm{p}<0.05 ; \dagger \mathrm{p}<0.01 ; \ddagger \mathrm{p}<0.001 v$ adults.

$\int \mathrm{p}<0.05 ; \uparrow \mathrm{p}<0.01 ; \| \mathrm{p}<0.001 v$ formula fed infants.

$\mathrm{ND}=$ not done. 
Table 4 Faecal SCFA concentration in faeces from infants either exclusively breast fed or exclusively formula fed

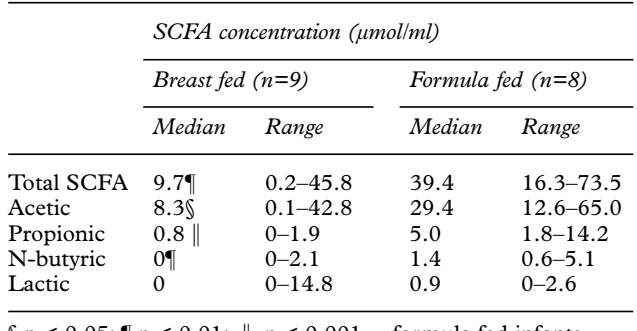

lactic acid with glucose, lactose, and raftilose than cultures of faeces from formula fed infants but this did not reach statistical significance because of the wide variations in amounts produced by individual infants. Six out of 11 cultures of faeces from breast fed infants with glucose produced more than $20 \mu \mathrm{mol} / \mathrm{ml}$ compared with $3 / 11$ cultures of faeces from formula fed infants, with $6 / 11$ cultures producing less than $10 \mu \mathrm{mol} / \mathrm{ml}$. Similarly with lactose, 6/11 cultures of faeces from breast fed infants cultures produced more than 30 $\mu \mathrm{mol} / \mathrm{ml}$ lactic acid compared with $2 / 10$ cultures of faeces from formula fed infants.

\section{FAECAL WATER}

Enough faecal material was available from 10 infants in each feeding group to determine faecal water content. There were wide variations in the proportion of faecal water in both groups. However, there were no significant differences in faecal water between breast fed infants $(31.4 \% \quad(21.7$ to 88.5$))$, and the formula fed infants $(29.9 \%$ (18.8 to 73.1$)$, median (range)).

FAECAL SCFA

The median concentrations of individual SCFA (except lactic acid) and total SCFA were significantly lower in breast fed infants than formula fed infants (table 4).

\section{Discussion}

The colonic microflora ferments unabsorbed carbohydrates producing SCFA. These are easily absorbed, promoting water absorption and preventing the osmotic diarrhoea which would otherwise result from the poor absorption of sugars in the colon. SCFA also provide a significant energy source, a mechanism referred to as 'colonic salvage'. 910

Differences in the microflora of breast fed and formula fed infants are well documented. The profile of faecal bacterial fermentation products also differs. The faeces of breast fed infants contain mainly acetic and lactic acids, whereas propionic acid replaces lactic acid in the faeces of formula fed infants and there is greater butyric acid production. The results of the present study confirm the differences between breast fed and formula fed infants seen in our earlier study of infants in Birmingham. ${ }^{3}$ In the Birmingham study, however, there was significantly more faecal lactic acid in breast fed infants than formula fed infants, which was not observed in this study in Glasgow.
In preweaning neonates, only milk-either breast or formula - is ingested. A significant amount of carbohydrate, mainly lactose, is likely to escape small intestinal digestion and absorption and enter the colon. The colonic bacteria may also ferment endogenous mucus and mucopolysaccharides as well as fructooligosaccharides in breast milk and substantial amounts of maltodextrins in some infant formula.

The SCFA produced during fermentation are determined by the substrates available, their fermentability, and the rate of breakdown. For example, lactic acid production is related to rapid and incomplete fermentation. The populations of each saccharolytic species are important. Breast fed infants have bifidobacteria as predominant organisms, whereas formula fed infants have more Enterobacteriaciae and Bacteroides spp. ${ }^{2}$ Host factors such as transit time, mucus secretion, and the presence of immunoglobulin and drugs may also affect fermentation. We do not know how these differ between breast fed and formula fed infants.

The microflora of breast fed and formula fed infants have developed in the presence of milk with lactose as the main carbohydrate. When new carbohydrates are included in the diet, they may influence composition and metabolic activity of the microflora. As the microflora of breast fed and formula fed infants differ substantially, both in bacterial populations and fermentation patterns, it might be expected that this would affect their ability to ferment new carbohydrates.

In this study, we found that the faecal bacteria of both groups of infants, breast fed and formula fed, were equally well able to ferment the sugars glucose and lactose, but they fermented the complex carbohydrates in soybean polysaccharide very poorly in comparison with adult faecal bacteria. Infants have fewer bacterial species in their colon than adults and their microflora are unlikely to have been exposed to the same range of unabsorbed carbohydrate. Thus the enzymes needed to ferment complex carbohydrates may not be present. SCFA absorption in the colon increases water absorption and reduces the risk of diarrhoea. In a study in adults, the ability of individuals to tolerate lactulose without an increase in stool output was decreased when antibiotics were given ${ }^{11}$ because of a decreased ability to produce the SCFA which would normally be absorbed, preventing osmotic diarrhoea. If formula fed infants had a lower fermentation capacity than breast fed infants this would contribute to their increased risk of diarrhoea. The results of our study, however, do not support this hypothesis.

In contrast to Lifshitz et al, ${ }^{6}$ we did not see a difference in SCFA production between breast fed and formula fed infants, although the final $\mathrm{pH}$ in our cultures containing sugars and oligosaccharide was similar to his low $\mathrm{pH}$ model for most individuals. The Lifshitz model used only a one hour incubation in contrast to our 24 hour model. The transit time of material through the colon, and therefore the time available for fermentation, is well in excess of 
one hour and thus any differences in lactose hydrolysis may be compensated for before there is any influence in stool output. The main finding of Liftshitz et $a l^{6}$ was that less lactose hydrolysis occurred in the formula fed infants, resulting in a greater potential osmolality due to lower fermentation at low $\mathrm{pH}$. We did not measure residual lactose in this study. However, it is likely that, apart from the complex carbohydrate, most of the added carbohydrate was fermented by 24 hours, increasing osmolality only by increasing SCFA production. In vivo these SCFA would be absorbed and have very little impact on luminal osmolality.

Although the formula fed and breast fed infants could both ferment sugars readily, the profile of SCFA produced in this study differed. The faecal flora of formula fed infants produced significantly more propionic acid with the sugars as substrates and significantly more n-butyric acid with glucose and raftilose than that of breast fed infants. This suggests that the acetic/lactic acid profile in faeces of breast fed infants and the acetic/propionic acid profile in faeces of formula fed infants is representative of events occurring during fermentation of sugars in the more proximal colon and is not due to differential absorption in the colon.

With complex carbohydrates there was a less marked difference between the breast fed and formula fed SCFA profiles, and the total amounts of SCFA produced were similar. This probably reflects the poor ability of both groups of infants to ferment the soybean polysaccharide and therefore their limited SCFA production.

The ability of neonates to ferment starch, another complex carbohydrate, was tested in vivo by Shulman et $a l^{12}$ using measurement of breath hydrogen. Infants of 4 weeks were fed corn cereal and the breath hydrogen produced was compared with that produced from lactulose. Cereal intake increased breath hydrogen, showing the inefficient digestion and absorption in the small intestine but also a significant fermentation capacity for complex carbohydrate in the colon. However, we have previously found significant amounts of unfermented starch in the faeces of young children up to 3 years of age, indicating that the fermentation capacity for complex carbohydrates develops gradually. ${ }^{13}$

In conclusion, we have shown that despite very different microflora, breast fed and formula fed infants appear to have similar fermentation capacity for simple sugars and oligosaccharides and are equally poor at fermenting soybean polysaccharide. It has been shown, however, that breast fed infants have a higher stool output than formula fed infants in terms of both frequency and stool weight, and this is likely to result in a higher capacity for fermentation. ${ }^{14} \mathrm{~A}$ faster transit, however, could reduce the time available for fermentation to reach completion and this may be reflected in the usually higher lactic acid content of the breast fed stool, ${ }^{3}$ although this was not found in the present study.

The different microflora produced characteristic profiles of SCFA which may have different physiological actions in the colon. Our results also confirm a low production of butyric acid in neonates. We have previously shown that faeces of breast fed infants contain almost no butyrate. ${ }^{3}$ Butyric acid is thought to be essential for the health of the colonic mucosa in adults. ${ }^{15}$ Either butyric acid is not as important as was previously believed or the colonic enterocytes of the neonate have different characteristics from those of the adult.

Although there was no difference in the fermentation capacity of formula fed and breast fed infants and no support for the hypothesis that a lower fermentation capacity of formula fed infants is a contributing factor to their increased risk of diarrhoea, the low ability of neonatal colonic bacteria to ferment complex carbohydrates is likely to increase the impact on stool output of complex carbohydrates introduced during early weaning. It is during weaning that breast fed infants are most susceptible to diarrhoea.

1 Howie PW, Forsyth JS, Ogston SA, Clark A, Florey CV. Protective effect of breast feeding against infection. $B M \mathcal{F}$ 1990;300:11-6.

2 Balmer SE, Wharton BA. Diet and faecal flora in the newborn: breast milk and infant formula. Arch Dis Child 1989;64:1672-7.

3 Edwards CA, Parrett AM, Balmer SE, Wharton BA. Faecal short chain fatty acids in breast-fed and formula-fed infants. Acta Paediatr 1994;83:459-62.

4 Adiotomre J, Eastwood MA, Edwards CA, Brydon WG. Dietary fibre; in vitro methods that anticipate nutrition and metabolic activity in humans. Am f Clin Nutr 1990;52:128-
mietary fibre; in vitro methods that anticipate nutrition and 34 .

5 Rasmussen HS, Holtug K, Ynggård C, Mortensen PB. Faecal concentrations and production rates of short chain fatty acids in normal neonates. Acta Paediatr Scand 1988;77: $365-8$

6 Lifschitz CH, Wolin MJ, Reed J. Characterisation of carbohydrate fermentation in faeces of formula fed and breast fed infants. Pediatr Res 1990;27:165-9.

7 Spiller GA, Chernoff MC, Hill RA, Gates JE, Nassar JJ, Shipley EA. Effect of purified cellulose, pectin and a low residue diet of faecal volatile fatty acids, transit time and faecal weight in humans. Am f Clin Nutr 1980;33:754-9.

8 Holdemann LV, Moore WEC, eds. Anaerobe laboratory manual. 2nd Ed. Blacksburg: Virginia Polytechnic Institute and State University, 1973.

9 Kien CL, Liechty EA, Mullett MD. Contribution of low-molecular weight compounds to the faecal excretion of carbohydrate energy in premature infants. Gastroenterology carbohydrate energy

10 Bond JH, Currier BE, Buchwald HL, Levitt MD. Colonic conservation of unabsorbed carohydrates. Gastroenterology 1980;78:444-7.

11 Rao SSC, Edwards CA, Austen C, Read NW, Holdsworth CD. Impaired colonic fermentation of carbohydrates after ampicillin. Gastroenterology 1988;94:928-32.

12 Shulman RJ, Wong WW, Irving CS, Nichols BL, Klein PD. Utilization of dietary cereal by young infants. 7 Pediatr 1983;103:23-8.

13 Verity K, Edwards CA. Resistant starch in young children [abstr]. Proc Nutr Soc 1994;53:105A.

14 Weaver LT, Ewing G, Taylor LC. The bowel habit of milk fed infants. F Paediatr Gastroenterol Nutr 1988;7:568-71.

15 Roediger WE. Utilisation of nutrients by isolated epithelial cells of rat colon. Gastroenterology 1982;83:424-9. 